\title{
Submillimeter Array Imaging of the Maser Emission from the $($ H3Olalphal) Radio Recombination Line in MWC 349A
}

\section{Citation}

Weintroub, Jonathan, James M. Moran, David J. Wilner, Ken Harbour Young, Ramprasad Rao, and Hiroko Shinnaga. 2008. "Submillimeter Array Imaging of the Maser Emission from the (H30 \alphal) Radio Recombination Line in MWC 349A." Astrophysical Journal 677 (2) (April 20): 1140-1150. doi:10.1086/529132.

\section{Published Version}

doi:10.1086/529132

\section{Permanent link}

http://nrs.harvard.edu/urn-3:HUL.InstRepos:11595848

\section{Terms of Use}

This article was downloaded from Harvard University's DASH repository, and is made available under the terms and conditions applicable to Other Posted Material, as set forth at http:// nrs.harvard.edu/urn-3:HUL.InstRepos:dash.current.terms-of-use\#LAA

\section{Share Your Story}

The Harvard community has made this article openly available.

Please share how this access benefits you. Submit a story.

Accessibility 
The Astrophysical Journal, 677:1140-1150, 2008 April 20

(C) 2008. The American Astronomical Society. All rights reserved. Printed in U.S.A.

\title{
SUBMILLIMETER ARRAY IMAGING OF THE MASER EMISSION FROM THE H30 $\alpha$ RADIO RECOMBINATION LINE IN MWC 349A
}

\author{
Jonathan Weintroub, James M. Moran, David J. Wilner, and Ken Young \\ Harvard-Smithsonian Center for Astrophysics, 60 Garden Street, Cambridge, MA 02138; jweintroub@cfa.harvard.edu \\ RAMPRASAD RAO \\ Institute of Astronomy and Astrophysics, Academia Sinica, 645 North A'ohoku Place, Hilo, HI 96720
}

AND

HiRoko SHINNAGa

Caltech Submillimeter Observatory, 111 Nowelo Street, Hilo, HI 96720

Received 2007 August 17; accepted 2008 January 5

\begin{abstract}
We used the Submillimeter Array to map the angular distribution of the $\mathrm{H} 30 \alpha$ recombination line $(231.9 \mathrm{GHz})$ in the circumstellar region of the peculiar star MWC 349A. The resolution was $1.2^{\prime \prime}$, but because of high signal-to-noise ratio we measured the positions of all maser components to accuracies better than $0.01^{\prime \prime}$, at a velocity resolution of $1 \mathrm{~km} \mathrm{~s}^{-1}$. The two strongest maser components (called high-velocity components) at velocities near -14 and $32 \mathrm{~km} \mathrm{~s}^{-1}$ are separated by $0.048^{\prime \prime} \pm 0.001^{\prime \prime}(60 \mathrm{AU})$ along a position angle of $102^{\circ} \pm 1^{\circ}$. The distribution of maser emission at velocities between and beyond these two strongest components were also provided. The continuum emission lies at the center of the maser distribution to within 10 mas. The masers appear to trace a nearly edge-on rotating disk structure, reminiscent of the water masers in Keplerian rotation in the nuclear accretion disk of the galaxy NGC 4258. However, the maser components in MWC 349A do not follow a simple Keplerian kinematic prescription with $v \sim r^{-1 / 2}$, but have a larger power-law index. We explore the possibility that the high-velocity masers trace spiral density or shock waves. We also emphasize caution in the interpretation of relative centroid maser positions where the maser is not clearly resolved in position or velocity, and we present simulations that illustrate the range of applicability of the centroiding method.
\end{abstract}

Subject headings: circumstellar matter - masers — radio lines: stars — stars: emission-line, Be stars: individual (MWC 349A) — stars: winds, outflows

\section{INTRODUCTION}

The hydrogen recombination line emission from the peculiar $\mathrm{B}$ [e] star MWC 349A is double-peaked at frequencies above $100 \mathrm{GHz}$, and is thought to be due to maser action in a gaseous rotating circumstellar disk. Thermal continuum emission from radio to IR wavelengths reveals that the star also has an ionized outflow. Maser action occurs in recombination lines from about $100 \mathrm{GHz}(\mathrm{H} 39 \alpha)$ to $1800 \mathrm{GHz}(\mathrm{H} 15 \alpha)$ and beyond (Strelnitski et al. 1996a, 1996b). A physical model developed by Thum et al. (1994a, 1994b) suggests that the maser spots originate on the ionized surface of a rotating disk that is nearly edge-on in orientation. Ponomarev et al. (1994) performed detailed radiative transfer modeling and concluded that the masers are probably unsaturated with a maximum negative opacity (gain) of about 5 .

High-resolution Very Large Array (VLA) continuum images of MWC 349A (e.g., White \& Becker 1985; Tafoya et al. 2004) show an hourglass-shaped image whose angular size scales with frequency $\nu$ as $\nu^{-0.7}$ and flux density as $\nu^{0.6}$, as expected for a dense ionized outflow. The star is located in the waist of an hourglassshaped gas structure, with the north-south lobes corresponding to outflows, and the putative rotating disk presumed to be located around the star and viewed nearly edge-on. It is interesting to note that VLA continuum images spanning the period of 1996-2006 (Rodríguez et al. 2007) show that the continuum morphology of the object has evolved from an hourglass structure to more of a rectangular projected structure (i.e., the waist of the hourglass structure is no longer prominent). The spectrum of the maser emission is also variable (see Martín-Pintado et al. 1989; Gordon et al. 2001).
Planesas et al. (1992) observed the H30 $\alpha$ maser emission and showed that its dominant characteristic is two features (aka "spots") at velocities of -14 and $32 \mathrm{~km} \mathrm{~s}^{-1}$ separated by about $0.065^{\prime \prime} \pm$ $0.005^{\prime \prime}$, along a position angle of about $107^{\circ} \pm 7^{\circ}$. The close correspondence between the position angle of the spots and that of the waist of the hourglass continuum map suggests that the maser spots are located in the waist of the continuum. For a distance of $1200 \mathrm{pc}$ (Cohen et al. 1985), the velocity and spatial separation between the two maser peaks of $47 \mathrm{~km} \mathrm{~s}^{-1}$ and $120 \mathrm{AU}$, respectively, and a central mass of about $30 M_{\odot}$, can be inferred from Kepler's third law (e.g., Strelnitski et al. 1996a).

IR continuum images at $1.65,2.27$, and $3.08 \mu \mathrm{m}$ were made by Danchi et al. (2001) with the Keck Telescope using an aperture masking technique. The images are elliptical with major axes of 36, 47, and 62 mas, respectively. The position angles of the images are about $100^{\circ}$. These images trace the dust and the neutral gas that is probably undergoing photoevaporation, giving rise to the whole hourglass ionized region (Hollenbach et al. 1994). The structures of the radio continuum at $7 \mathrm{~mm}$ ( Tafoya et al. 2004), the IR at $2.27 \mu \mathrm{m}$, and the maser emission from OVRO (Owens Valley Radio Observatory; Planesas et al. 1992) are shown in a composite image in Figure 1.

Here we present interferometric images of the maser emission in the $\mathrm{H} 30 \alpha$ radio recombination line, obtained with the Submillimeter Array (SMA), ${ }^{1}$ which have much higher signal-to-noise

\footnotetext{
1 The Submillimeter Array is a joint project between the Smithsonian Astrophysical Observatory and the Academia Sinica Institute of Astronomy and Astrophysics and is funded by the Smithsonian Institution and the Academia Sinica.
} 


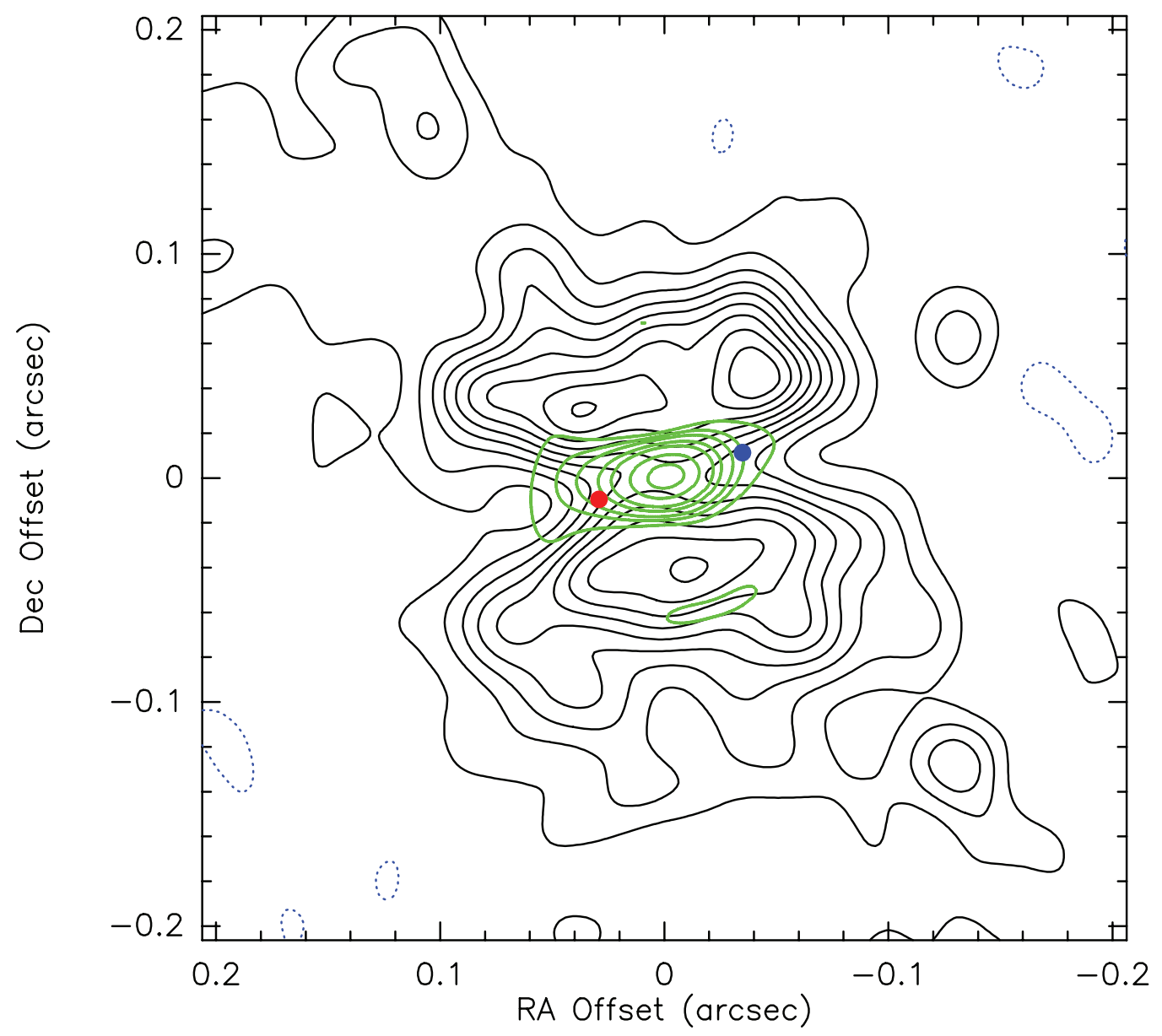

FIG. 1.- Superposition of the $7 \mathrm{~mm}$ VLA continuum image (black contours) made by Tafoya et al. (2004), $2.27 \mu \mathrm{m}$ Keck aperture-masked image (green contours) made by Danchi et al. (2001), and two maser components (red dot, $32 \mathrm{~km} \mathrm{~s}^{-1}$; blue dot, $-16 \mathrm{~km} \mathrm{~s}^{-1}$ ) measured by Planesas et al. (1992). Relative astrometric accuracy among the wavelength bands was poorer than $0.1^{\prime \prime}$; we have coregistered the images to maximize the probable symmetry of the source. This figure is an updated version of a similar figure published by Danchi et al. (2001). The reference position for the image is R.A. $=20^{\mathrm{h}} 32^{\mathrm{m}} 45.528^{\mathrm{s}}$ and decl. $=40^{\circ} 39^{\prime} 36.622^{\prime \prime}(\mathrm{J} 2000.0)$, taken from the VLA observations.

ratios (S/Ns) than those of previous investigations. This allows for the study of the distribution of maser emission in significantly greater detail. We have additional data from the SMA in the $\mathrm{H} 26 \alpha(353 \mathrm{GHz})$ and $\mathrm{H} 21 \alpha(652 \mathrm{GHz})$ transitions. These results have lower $\mathrm{S} / \mathrm{Ns}$ than the ones presented here. They will be discussed in a later publication.

\section{OBSERVATIONS AND DATA REDUCTION}

The $\mathrm{H} 30 \alpha$ observations made on 2004 September 12 are presented in this paper. The weather was excellent for $230 \mathrm{GHz}$ operations with $\tau_{225}$ varying between 0.05 and 0.07 during the night. The array was in the "extended" configuration with seven antennas in operation (pads $1,8,11,14,15,16$, and 17) providing baselines from 21 to $156 \mathrm{~m}$. The synthesized beam at $230 \mathrm{GHz}$ was $1.2^{\prime \prime} \times 0.9^{\prime \prime}$ with a P.A. of $90^{\circ}$. The correlator was configured in a hybrid mode. One $82 \mathrm{MHz}$ "chunk" was centered on the maser emission and fed to a 512-channel cross correlator. The other 23 chunks were set at frequencies surrounding the maser band and fed to a bank of 32-channel correlators. The spectral resolution on the maser chunk was formally $0.24 \mathrm{MHz}$, but the data were averaged into bins at intervals of $0.76 \mathrm{MHz}$, exactly $1 \mathrm{~km} \mathrm{~s}^{-1}$, covering a velocity range of -53 to $53 \mathrm{~km} \mathrm{~s}^{-1}$.

The observing schedule interspersed observations of MWC $349 \mathrm{~A}$ with the quasar calibrators $2013+370,2202+422$, and
$2232+117$. The SMA correlator was configured to take $30 \mathrm{~s}$ scans. Our observing cycle consisted of 24 scans on MWC 349A, followed by 6 scans on each of the three quasar calibrators. $2232+$ 117 became available a little later than the other two quasars, and was the only quasar calibrator in the sky at the end of the track. After MWC 349A set, we took 50 minutes of data on Titan for absolute flux calibration. The total integration time on MWC 349A was $4.8 \mathrm{hr}$.

Bandpass calibration was a crucial aspect of these observations, since the angular distribution of the maser emission is only about $0.05^{\prime \prime}$, or one-twentieth of the synthesized beam. Hence, after MWC 349A set, over $3 \mathrm{hr}$ of additional bandpass calibration data on Venus and Uranus was taken. We tested the stability of our bandpasses by dividing the Venus data sets into two parts and calibrating one half with the other. We then did a vector average of the visibility spectra on all baselines in the calibrated portion of the data. The phase deviation from zero was less than $\pm 0.5^{\circ}$ across the band. This means that any systematic error in relative positions is less than $1 / 720$ of the resolution, or about $\pm 0.001^{\prime \prime}$.

Data reduction was carried out with the software packages MIR (for flagging, bandpass, gain, and flux calibration) and Miriad (for inverting the visibilities to form line images and applying the CLEAN algorithm). In MIR, data were first weighted by system temperature to account for sensitivity variations due to elevation 

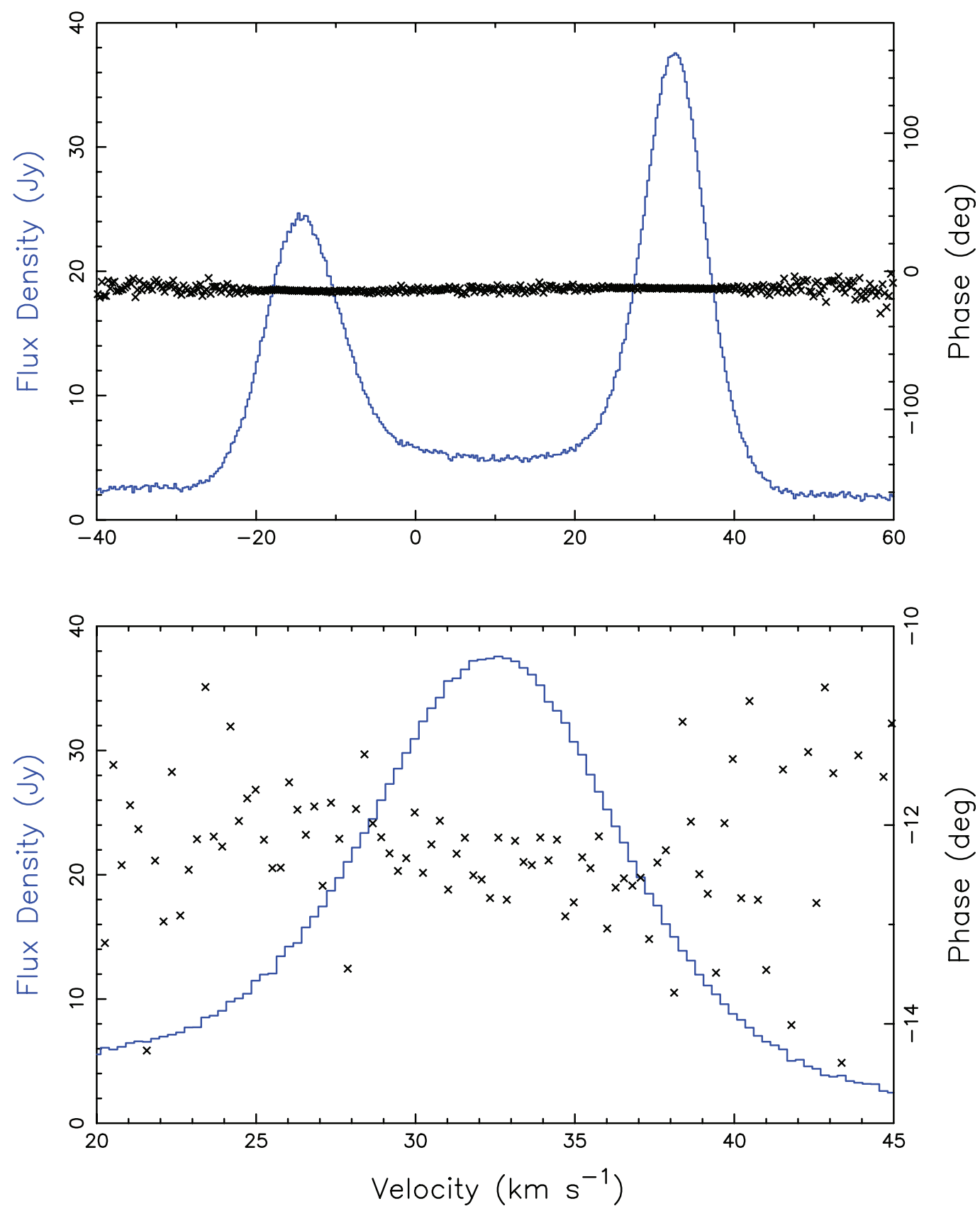

FIG. 2.-Top: Vector averaged visibility spectrum of all of the data on all baselines from the 2004 September 12 observations of the H30 $\alpha$ line; bandpass, gain, and flux calibrations have been applied. Bottom: Detailed view of the visibility spectrum in the redshifted part of the spectrum. The scatter in the phase across the red feature is small because of the high $\mathrm{S} / \mathrm{N}$ and angular proximity of the spectral components. In both panels, crosses show the phase, and the continuous lines show the amplitude. The velocity axis refers to the local standard of rest and is based on a rest frequency of $231.9009 \mathrm{GHz}$. In this figure only the data are displayed at the intrinsic correlator resolution of $0.24 \mathrm{~km} \mathrm{~s}^{-1}$.

and atmospheric changes. The bandpass calibration used all three quasars and $3 \mathrm{hr}$ of high $\mathrm{S} / \mathrm{N}$ data from Venus. The gain calibration used the interspersed data from the quasar 2013+370. Absolute flux calibration was achieved on the data from Titan, whose flux density was estimated with the SMA's online visibility calculator as $1.2 \mathrm{Jy}$. The MWC 349A data were exported in FITS file format for further manipulation in Miriad. The latter program was used to invert the visibilities to create line and continuum images. The Miriad implementation of the CLEAN algorithm was used to deconvolve the dirty beam from the images. The vector- averaged visibility spectrum is shown in Figure 2 . The rms accuracy of our images at $1 \mathrm{~km} \mathrm{~s}^{-1}$ resolution was about $125 \mathrm{mJy}$.

\section{ASTROMETRY AND CONTINUUM MEASUREMENT}

When making these observations, the phase center of the array was set to the published coordinates of MWC 349A from the SIMBAD database: R.A. $=20^{\mathrm{h}} 32^{\mathrm{m}} 45.53^{\mathrm{s}}$ and decl. $=$ $40^{\circ} 39^{\prime} 37.0^{\prime \prime}(\mathrm{J} 2000.0)$. These coordinates came from the catalog of $\mathrm{H} \alpha$ emission stars in the northern Milky Way published by Kohoutek \& Wehmeyer (1999). The coordinates we 
derive for the centroid of the $1.3 \mathrm{~mm}$ continuum emission are R.A. $=20^{\mathrm{h}} 32^{\mathrm{m}} 45.53^{\mathrm{s}} \pm 0.01^{\mathrm{s}}$ and decl. $=40^{\circ} 39^{\prime} 36.6^{\prime \prime} \pm 0.1^{\prime \prime}$ (J2000.0). There is significant discrepancy of $0.4^{\prime \prime}$ in declination. Our position agrees well with the centroid position of the $7 \mathrm{~mm}$ continuum emission determined by Rodríguez et al. (2007) of R.A. $=20^{\mathrm{h}} 32^{\mathrm{m}} 45.528^{\mathrm{s}} \pm 0.05^{\mathrm{s}}$ and decl. $=40^{\circ} 39^{\prime} 36.622^{\prime \prime} \pm$ $0.005^{\prime \prime}$ (J2000.0) for epoch 2004.9. Since the positions of the radio images are coincident to within their errors $0.1^{\prime \prime}$, we suspect that the apparent offset of the $\mathrm{H} \alpha$ position in declination is due to uncertainty in the optical measurement. All images in this paper are referenced to the $230 \mathrm{GHz}$ position of the continuum emission.

The spectrum of MWC 349A closely follows the form of $\nu^{0.6}$, and the angular size proportional to $\nu^{-0.7}$, indicative of a square law decrease in density with radius. The flux density and size extrapolated from 43 to $230 \mathrm{GHz}$ are $2.1 \mathrm{Jy}$ and $0.04^{\prime \prime}$, respectively. The flux density we measured was about 2 Jy. The source was unresolved, as expected, by the SMA. The relative alignment of the continuum and maser emission is accurate to $0.01^{\prime \prime}$.

\section{RESULTS}

We assumed that the image at each velocity could be modeled as a point source. To determine the centroid position, a twodimensional Gaussian function was fitted to each image. A discussion of systematic errors that can result from this technique due to multiple unresolved features is described in $\S 5.4$. Briefly, we found that if the spectral widths of individual masers are appropriate for unsaturated amplification with a gain of about 100 or more, then the position centroiding analysis (PCA) method will give unbiased results. If, however, the high-velocity emission near -14 and $32 \mathrm{~km} \mathrm{~s}^{-1}$ is dominated by single components with amplification gain factors of only about 5 , then the distribution of components around the velocities will be biased and the inferred power-law indices will be larger than the Keplerian value of 0.5 .

The formal error in the position estimate derived by the PCA method is given by the equation

$$
\Delta \theta=\frac{1}{2} \frac{\theta_{r}}{\mathrm{~S} / \mathrm{N}}
$$

where $\theta_{r}$ is the angular resolution (beamwidth) of the array and $\mathrm{S} / \mathrm{N}$ is the signal-to-noise ratio in the imaged velocity channel. Hence, the accuracy of the PCA method can be much greater than the beamwidth for high values of $\mathrm{S} / \mathrm{N}$. For the strongest feature of $42 \mathrm{Jy}$, with rms noise of $125 \mathrm{mJy}$ and resolution of $1.2^{\prime \prime}$, the formal centroiding accuracy is $0.0018^{\prime \prime}$. The spectrum in Figure 2 (bottom) shows that the rms phase noise is about $1^{\circ}$. This is about an order of magnitude better than the accuracy achieved by Planesas et al. (1992).

Figure 3 shows the spectrum and the position associated with each velocity channel from the centroiding analysis. Note how the right ascension trends downward in the blueshifted component, reverses and trends upward in the trough between the components, then reverses and trends downward again through the redshifted peak. No such reversals are apparent in the corresponding declination plot.

Figure 4 shows the distribution of masers as a function of velocity (aka a "spot" map). Note that every velocity channel with significant flux density is shown. No attempts have been made to identify specific spectral features. Figure 4 also shows a positionvelocity $(p-v)$ plot of velocity versus right ascension, approximately the major axis of the distribution. In the discussion that follows, we refer to the emission in the spectral peaks (i.e., $<0$ and $>20 \mathrm{~km} \mathrm{~s}^{-1}$ ) as "high-velocity" emission, and the spectral range inbetween where the position changes linearly with position as the "low-velocity" emission.
We can compare our results quantitatively with those of Planesas et al. (1992). They measured the mean separation between emission in the range $32 \pm 10 \mathrm{~km} \mathrm{~s}^{-1}$ and that in the range $-16 \pm 10 \mathrm{~km} \mathrm{~s}^{-1}$ to be $0.065^{\prime \prime} \pm 0.005^{\prime \prime}$ at a position angle of $107^{\circ} \pm 7^{\circ}$. If we average our measurements over the same range (amplitude weighted) we obtain a separation of $0.048^{\prime \prime} \pm 0.001^{\prime \prime}$ at a position angle of $102^{\circ} \pm 1^{\circ}$.

\section{DISCUSSION}

We discuss possible dynamical models to explain the positionvelocity data that we have obtained. We begin by reviewing the characteristics of a Keplerian disk to frame the discussion. We find that the high-velocity features follow a power-law distribution of the form $v \sim r^{-\alpha}$, where $\alpha$ is much larger than the Keplerian index of 0.5 . We then show that the large power-law index can be understood if the masers are in Keplerian motion, but distributed along spiral arms. Finally, we present simulations to show the reliability of the centroiding method of determining relative maser positions to small fractions of the spatial resolution.

\subsection{A Thin Annular Keplerian Disk}

Figure 5 shows the characteristic $p$ - $v$ relationship of a set of discrete masers in purely Keplerian rotation. For an edge-on disk full of compact masing sources in Keplerian rotation, a maser spot at radius $r$ has a velocity $v$ and a line-of-sight velocity $v_{z}$ of (see Fig. 5)

$$
v_{z}=v \sin \theta=\sqrt{\frac{G M}{r}} \sin \theta,
$$

where $G$ is the gravitational constant, $M$ is the stellar mass, and $b$ is the projected offset from the star or impact parameter. Since $\sin \theta=b / r, v_{z}$ will be proportional to $b$ for masers at fixed values of $r$, i.e.,

$$
v_{z}=\sqrt{\frac{G M}{r^{3}}} b .
$$

In practical units equation (3) can be written as $v_{z}=27\left(\mathrm{M} / \mathrm{r}^{3}\right)^{1 / 2} b$ for a distance of $1200 \mathrm{kpc}$ when $v_{z}$ is in $\mathrm{km} \mathrm{s}^{-1}, b$ and $r$ are in milliarcseconds, and $M$ is in $M_{\odot}$. Masers at a fixed radius will extend along a straight line between the Keplerian limits where $b= \pm r$. For a disk with maser emission bounded by an inner and outer radii of $r_{i}$ and $r_{o}$, the masers will lie within a "bowtie" region in the $p$ - $v$ diagram, bounded by the straight lines defined by $v_{z}=$ $\left(G M / r_{i}^{3}\right)^{1 / 2} b$ and $v_{z}=\left(G M / r_{o}^{3}\right)^{1 / 2} b$, respectively (see Fig. 5). Features lying along the curved outer boundaries arise on the "midline," where $\theta= \pm 90^{\circ}$.

The distribution of masers in the actual $p-v$ diagram suggests the masers are in three regions: approximately along the midline, one region on either side of the star, and in a relatively thin annular region of radius equal to about the outer radius $\left(0.025^{\prime \prime}\right.$, or about $30 \mathrm{AU}$ ) as traced by the high-velocity masers. It is not possible to tell whether masers in this annular region come from the front side or back side of the disk. However, if they are spread out on both sides, then the ratio of the minor to the major axis, which is about 0.25 , suggests that the disk is tilted by about $15^{\circ}$ to the line of sight. If the front side of the disk is tipped down, as suggested by Rodríguez \& Bastian (1994) with respect to us, there appears to be a deficit of masers on the front side of the disk (see Fig. 4). The lower panel of Figure 4 shows the $p$ - $v$ plot with Keplerian curves for masses of 10, 20, and $30 M_{\odot}$. The actual distribution of masers in the diagram crosses these three curves; hence, it is difficult to assign a specific mass without a more complex model (see $\S 5.4$ ). 

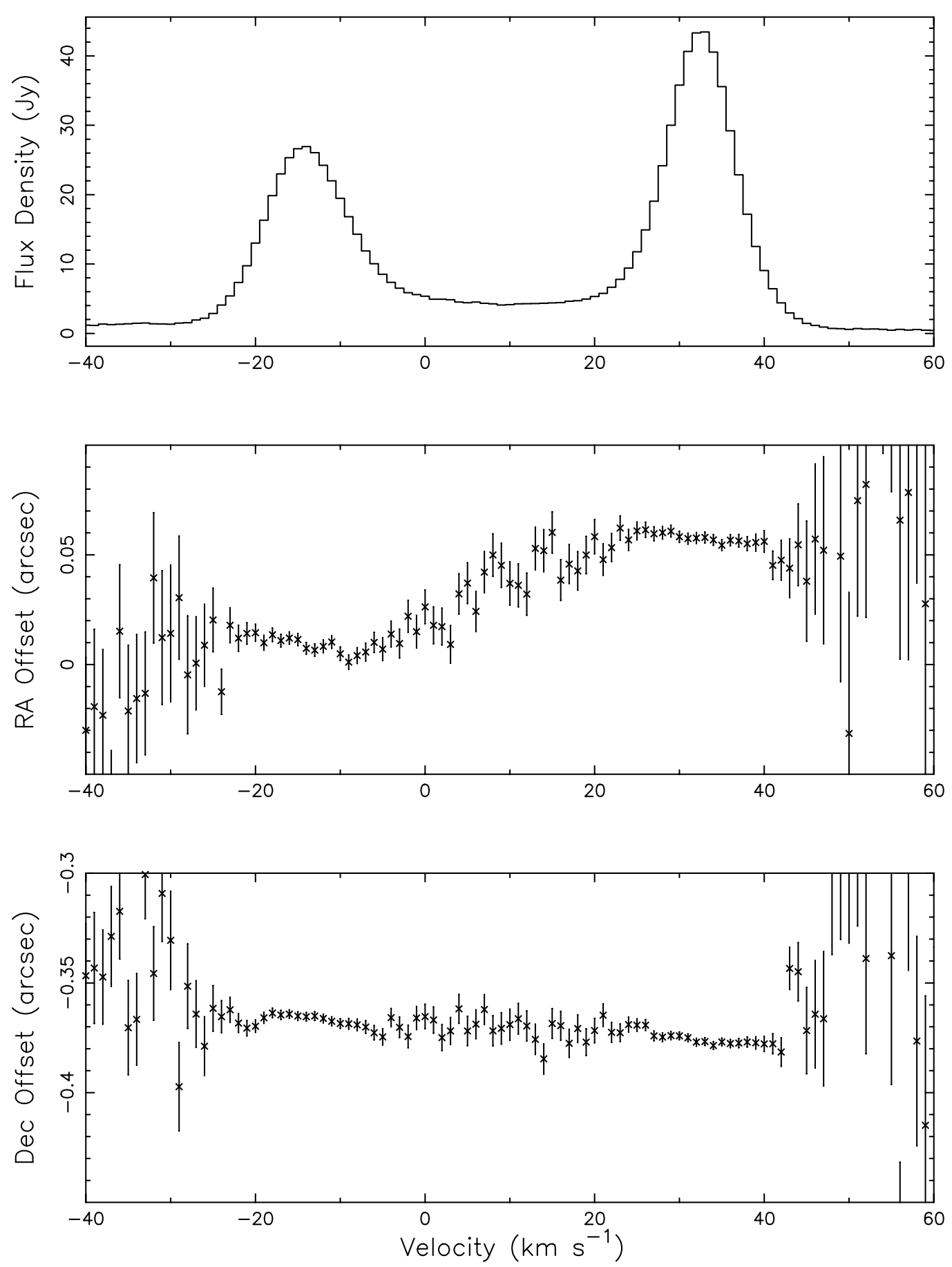

FIG. 3.-Results of the centroiding analysis applied to each velocity channel. Top: Spectrum, i.e., the peak amplitude at each velocity. Middle and bottom: Right ascension and declination positions at each velocity with respect to the phase center of the array. The continuum source is at relative position: $\Delta \mathrm{R} . \mathrm{A} .=0.031^{\prime \prime}$ and $\Delta$ decl. $=$ $-0.369^{\prime \prime}$. The position errors shown are based on the PCA method, and closely approximated by eq. (1).

\subsection{A Power-Law Velocity Curve}

We can characterize the "Keplerian" wings by a power-law model of the form

$$
v_{z}(b)=v_{0}+k\left(b-b_{0}\right)^{-\alpha} .
$$

We have used the right ascension positions for $b$. Since this equation is nonlinear in the parameters, we solved for their values through a $\chi^{2}$ minimization technique. The errors were estimated from the offset from the global minimum necessary for $\chi^{2}$ to increase by 1 . The parameters and their errors are:

$$
\begin{aligned}
v_{0} & =2 \pm 3 \mathrm{~km} \mathrm{~s}^{-1}, \\
b_{0} & =0.005^{\prime \prime} \pm 0.002^{\prime \prime}, \\
k & =0.00014 \pm 0.00003, \\
\alpha & =3.2 \pm 0.1 .
\end{aligned}
$$



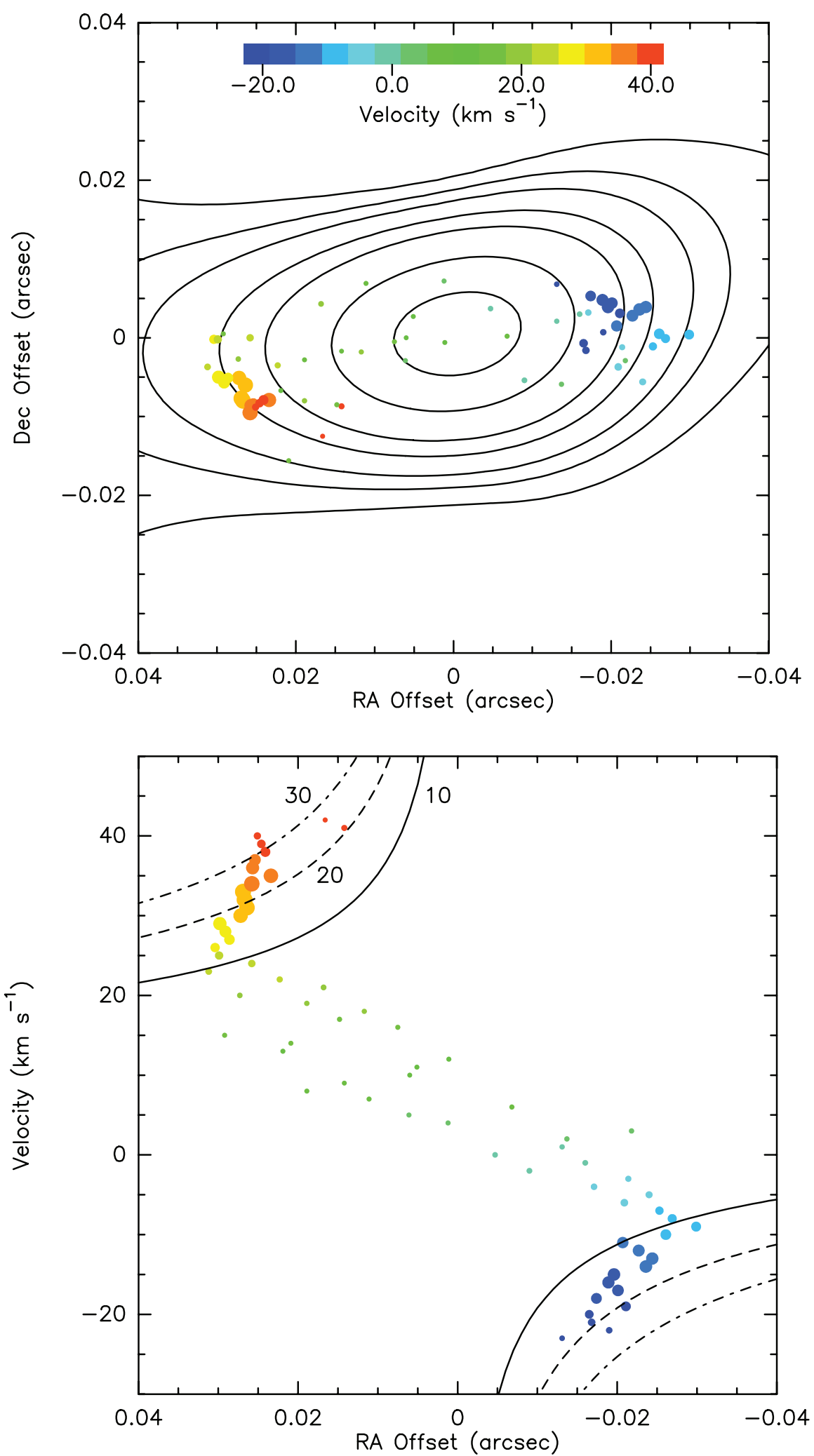

FIG. 4.-Top: Distribution of maser spots with respect to the position of the unresolved $230 \mathrm{GHz}$ continuum emission. This relative alignment is accurate to $0.01^{\prime \prime}$. An angular offset of $0.05^{\prime \prime}$ corresponds to a linear dimension of $60 \mathrm{AU}$ or $0.9 \times 10^{15} \mathrm{~cm}$ for a distance of $1200 \mathrm{pc}$. Areas of the spots are proportional to their flux densities and color coded by velocity. Error bars, shown in Fig. 3, are omitted here for clarity. Contours represent the $2.27 \mu \mathrm{m} \mathrm{IR}$ emission from Danchi et al. (2001). The IR image is nominally aligned with the continuum position. Bottom: The $p-v$ diagram, where right ascension is used as the position axis. High-velocity data points (velocities in the range $21-42$ and -5 to $-21 \mathrm{~km} \mathrm{~s}^{-1}$ ) are overlaid with Keplerian curves for central masses of 10,20 , and $30 M_{\odot}$. The high-velocity wings are considerably steeper than expected for Keplerian motion at a fixed azimuth angle. 


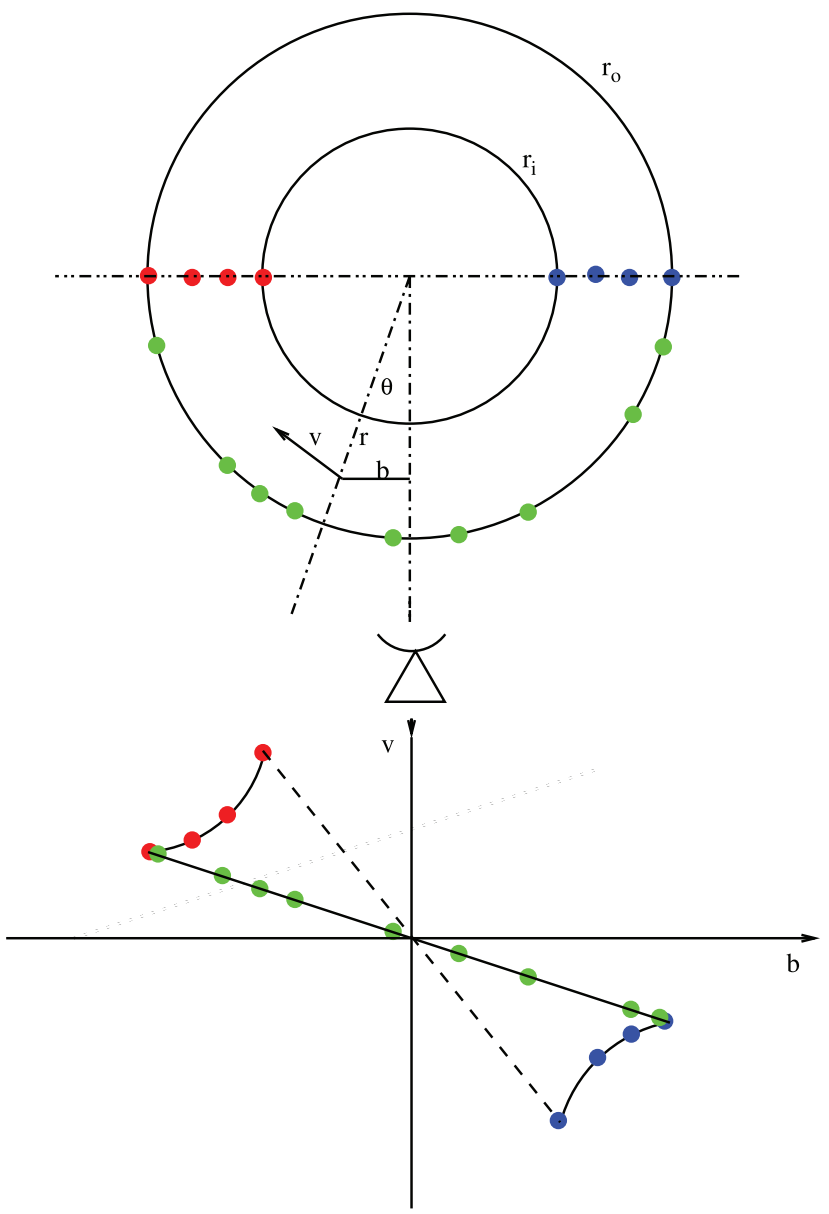

FIG. 5.- Sketch showing the maser positions within an edge-on unfilled disk in Keplerian rotation. The small filled circles represent the masers. Their positions are shown within the disk, and corresponding positions on the $p$ - $v$ diagram. Were the masers randomly distributed, they would randomly fill the characteristic "bowtie" envelope; however, the shape of the measured $p$ - $v$ diagram of Fig. 4 suggests that the masers are concentrated on the outer edge of the disk, with the curved Keplerian segments corresponding to the masers along the midline (the line passing through the star at the center of the torus, and perpendicular to the line of sight).

The fitted power-law rotation curve is shown on the $p$ - $v$ data in Figure 6 . A bowtie diagram has been superimposed whose linear limits have been set according to the limits of the high-velocity features. Note that the blueshifted features have more curvature in the $p$ - $v$ diagram than the redshifted features. Because of the symmetry imposed in the fit (i.e., both sides of the $p$ - $v$ diagram are assumed to have the same power-law index), the centroid of the $p$ - $v$ diagram is moved to the apparently off-centered location as shown. The systemic velocity is $2 \mathrm{~km} \mathrm{~s}^{-1}$ in this model, significantly offset from the mean velocity of the maser emission of $9 \mathrm{~km} \mathrm{~s}^{-1}$ and the central position is $0.005^{\prime \prime}$ offset from the continuum peak. The masers lie in the angular region of radii of about $0.019^{\prime \prime}$ and $0.032^{\prime \prime}$. Note, however, that the outer edge is defined by the blueshifted features, while the inner edge is defined by the redshifted features, and neither side of the disk is completely filled.

This model seems somewhat unrealistic due to the irregular boundaries of the disk and unusual systemic velocity. Positional symmetry could be restored if the power-law indices for the red and blue features were allowed to be different. The power-law model is unusual, since the power-law index is so large. A possible explanation could be that the disk is undergoing a magnetically powered spin-up. Thum \& Morris (1999) measured the line-of- sight magnetic field strength in the masing gas to be $22 \mathrm{mG}$. If the masers lie on the midline of a rotating disk, this field would correspond to a toroidal component. Even if the mass of the disk is assumed to be $30 M_{\odot}$, the kinetic energy is considerably less than both magnetic and thermal energy; hence, the magnetic field may control the dynamics.

\subsection{A Spiral Arm Model}

The velocities of the high-velocity maser components may be consistent with Keplerian motion if they do not lie on a line of constant azimuth angle such as $\theta= \pm 90^{\circ}$ (i.e., midline). We consider the case where the maser velocities are Keplerian, but the values of $v_{z}$ deviate substantially because of the distribution of azimuth angles. We assumed that the disk is edge-on and that the central stellar mass is $30 M_{\odot}$. Note that the $p$ - $v$ diagram for a $30 M_{\odot}$ star falls outside the $p-v$ data and just touches it at extreme velocities (see Fig. 4). The azimuth angle of any maser with position $b$ and line-of-sight velocity $v_{z}$ from equation (2) will be given by

$$
\theta= \pm \sin ^{-1}\left(\frac{b v_{z}^{2}}{G M}\right)^{1 / 3}
$$

The position with respect to the midline, $z$, is then given by $z=$ $b / \tan \theta$. Note that there is a twofold degeneracy in this calculation, i.e., we cannot determine whether a particular maser is on the front side or back side of the midline from its velocity alone. We have chosen to put the red features on the near side of the disk, in order to allow a trailing spiral arm configuration. Note that a careful examination of Figure 4 shows there is a slight, but significant, trend for both the red and blue features to increase in declination as their velocities decrease. In order for this to be consistent with the spiral sense, the disk must be tipped up in front (i.e., $i=85^{\circ}$ ) and have a position angle (P.A.) of about $115^{\circ}$. Note that the apparent P.A. of the masers is about $102^{\circ}$. The large-scale ionization structure suggests that the inclination angle is $95^{\circ}$ (disk tipped down in front; Rodríguez \& Bastian 1994), and the P.A. of the dust is about $100^{\circ}$. These orientations would mean that the maser disk is misaligned with the dust structure and the largescale structure of the ionized gas by about $10^{\circ}$ in both P.A. and $i$. An alternative model would be that the masers are tracing a leading spiral structure. The P.A. would still need to be about $115^{\circ}$, but the inclination angle would be $95^{\circ}$ (tipped down in front). We note that the spiral arms in most spiral galaxies are trailing. However, there are a few known examples of leading spiral arms (e.g., NGC 4622; Buta et al. 2003). We favor the trailing spiral model with the front side of the disk tipped up. We selected a spiral structure of the form $r=r_{0} e^{k(\theta-\pi / 2)}$ to approximate the maser distribution. The fitted spiral model is shown in Figure 7.

We will not explore or attempt to justify the existence of a spiral structure in the envelope of MWC 349A. However, we mention that Quillen et al. (2005) have developed a theory of spiral arms to explain the appearance of the circumstellar dust disks of HD 100546 and HD 141569. Note, however, that the Toomre $Q$-parameter associated with the ionized disk of MWC 349A is much greater than unity because the surface density of the ionized portion of the disk is so small. Hence, self-gravity is not a likely explanation for spiral structure. That is, $Q=v_{s} \Omega /(G \Sigma)$, where $v_{s}$ is the sound speed $\left(\sim 10 \mathrm{~km} \mathrm{~s}^{-1}\right), \Omega$ is the angular rotation rate (for $r=4 \times 10^{14} \mathrm{~cm}$ and $v=32 \mathrm{~km} \mathrm{~s}^{-1}, \Omega=5 \times 10^{-9} \mathrm{~s}^{-1}$ ), and $\Sigma$ is the surface density (for $n_{e}=10^{7} \mathrm{~cm}^{-3}$ and thickness of $10^{14} \mathrm{~cm}, \Sigma=2.5 \times 10^{-3} \mathrm{~g} \mathrm{~cm}^{-2}$ ). However, it may be possible that the stellar companion MWC 349B, which is offset by $2.4^{\prime \prime}$ at 


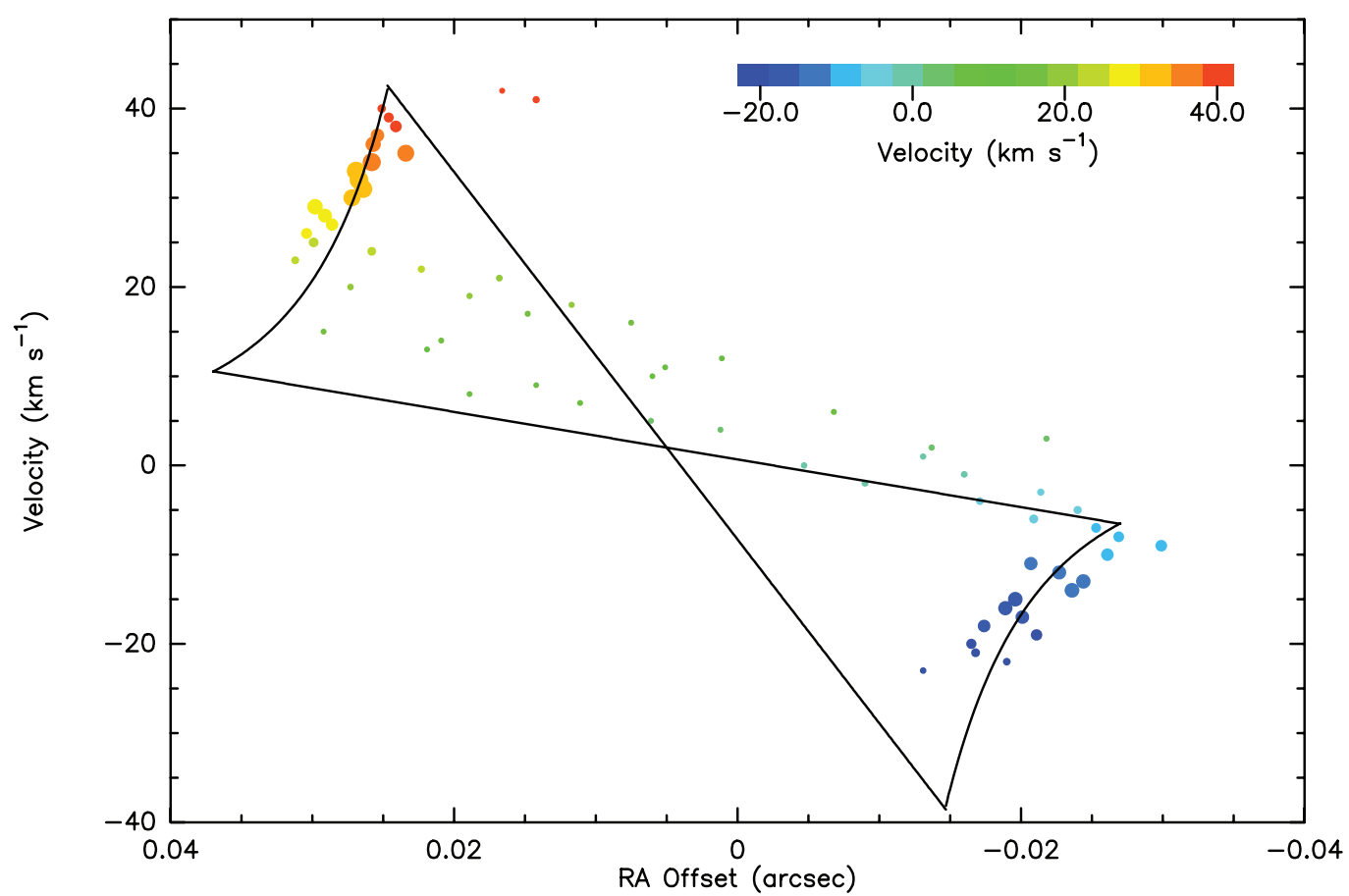

Fig. 6.-Power-law model fit to the high-velocity portions of the $p$ - $v$ data. Straight lines of the bowtie have been set to match the limits of the high-velocity emission and correspond to radii of $0.019^{\prime \prime}$ and $0.032^{\prime \prime}$, respectively. The fact that some features lie outside the bowtie area could be an indication of the thickness of the disk or its inclination.

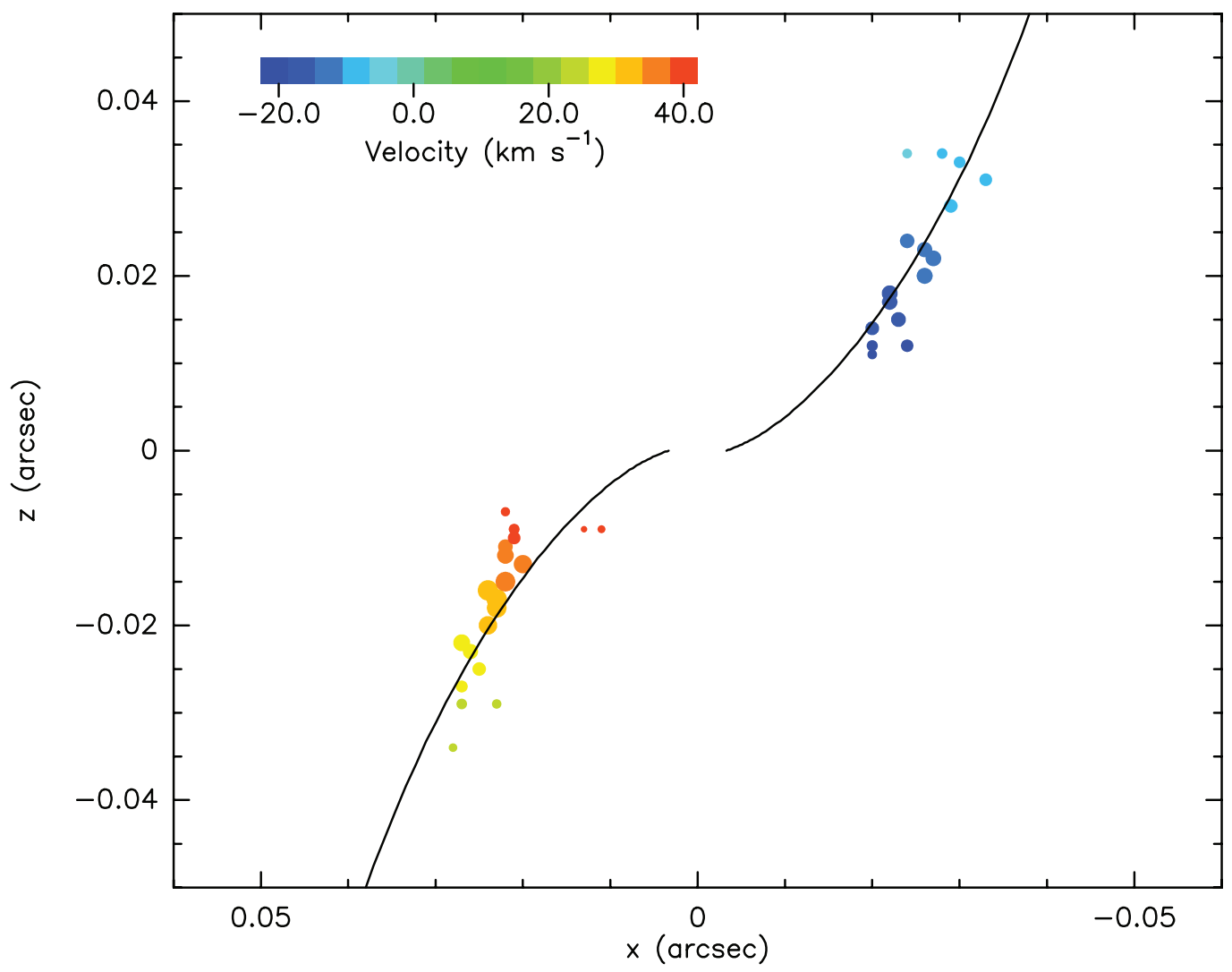

FIG. 7.- "Top" view of the maser distribution, i.e., seen from an axis perpendicular to the plane of the maser distribution. The direction to the earth is along the negative $z$-axis. The deprojection was accomplished by assuming that masers are in Keplerian orbits with the use of eq. (6). The redshifted features and the blueshifted features were assumed to arise from in front of and behind the midline, respectively. The rapid change in azimuth angle with velocity can explain the steepness of the $p$ - $v$ plot. A possible model with trailing arms is plotted on the data. This model requires that the P.A. of the disk be about $115^{\circ}$ (see Fig. 4) and that the inclination be about $85^{\circ}$ in order to explain the declination distributions of the masers. 
a P.A. of $100^{\circ} \pm 2^{\circ}$ (Cohen et al. 1985) (approximately at the P.A. of the maser disk), could tidally disrupt the disk.

\subsection{Evaluation of the Applicability of the Position Centroid Analysis (PCM) Method}

The technique of centroiding images to obtain positional measurements with accuracies much smaller than the instrumental resolution is well established in all wavelength regimes in astronomy. For an isolated source, with no confounding systematic measurement problems, the position can be found to the accuracy imposed by the instrumental noise limit (i.e., eq. [1]). This technique has been applied with particular effect in determining distributions of maser sources where the masing components are clearly distinguishable in both velocity and position (see, for example, Reid et al. 1980). In this case each maser feature, which occupies a unique velocity range, appears as an unresolved spot. Even in the case where the features are well resolved in velocity only, but not in position, this technique is very effective. For example, in NGC 4258, the features are well separated in velocity over a range of $2000 \mathrm{~km} \mathrm{~s}^{-1}$ with line widths of about $1 \mathrm{~km} \mathrm{~s}^{-1}$, but not angular extent where the angular extent for the low-velocity masers is 1 mas and the instrumental resolution is about $200 \mu$ as (see Moran et al. 1999).

In the case of MWC 349A, the maser spectrum has a rather simple appearance, dominated by two almost perfectly Gaussianshaped profiles centered at 32 and $-14 \mathrm{~km} \mathrm{~s}^{-1}$. Although the instrumental resolution of the SMA is much finer than the line widths of these features, their profiles do not reveal any substructure. Hence, the maser is resolved neither in velocity nor position, and the interpretation of centroided images must be made with caution and insight. Systematic artifacts, such as the appearance of a velocity gradient in a source that is actually composed of two components closely spaced in velocity and position, is well known. For example, Chen et al. (2006) have shown that a source of molecular emission (nonmaser) in $\mathrm{W} 3\left(\mathrm{H}_{2} \mathrm{O}\right)$, thought to be a gradient indicative of rotational motion, was in fact a double source whose components have slightly different velocities.

In this section, we report the results of the simulations we have made for MWC 349A following the methodology of Chen et al. (2006) to investigate the effects of source blending. Since the structure we have discussed in this paper is essentially one-dimensional (i.e., along a line with P.A. of about $102^{\circ}$ ), we have done only a simulation in one positional coordinate. We generated data for an image "square," i.e., intensity versus velocity and position coordinate $(x)$ from a set of Gaussian components. We then fit the image at each velocity with a Gaussian function and estimate its central position. Note that the width of the Gaussian function in position is always very close to the instrumental beamwidth (1.2" in our case), since the total maser extent is only $0.06^{\prime \prime}$.

A critical characteristic of the MWC 349A spectrum is the broad and flat component between the two distinct Gaussian peaks. Our simplest model consisted of three components, the two relatively narrow components at 32 and $-14 \mathrm{~km} \mathrm{~s}^{-1}$ and a very broad component at the systemic velocity of $9 \mathrm{~km} \mathrm{~s}^{-1}$ (Gordon [2003] has made a similar spectral decomposition). The parameters of this model are listed in Table 1. The simulated spectrum is shown in Figure 8 with the actual spectral data superimposed. The central component must be broad enough in velocity to simulate the flat appearance near $9 \mathrm{~km} \mathrm{~s}^{-1}$, but not so broad as to extend beyond the outer components. Even with the large width of $21 \mathrm{~km} \mathrm{~s}^{-1}$, the model spectrum is not as flat as the data in the central region (see Fig. 8, bottom left). The centroids determined from the simulated images as a function of velocity are shown in Figure 8. What happens effectively is that each component "controls"
TABLE 1

Parameters of Three- and Nine-Component Simulations

\begin{tabular}{|c|c|c|c|}
\hline $\begin{array}{l}\text { Amplitude } \\
\text { (Jy) }\end{array}$ & $\begin{array}{c}\text { Velocity } \\
\left(\mathrm{km} \mathrm{s}^{-1}\right)\end{array}$ & $\begin{array}{c}\text { Width }^{\mathrm{a}} \\
\left(\mathrm{km} \mathrm{s}^{-1}\right)\end{array}$ & $\begin{array}{l}\text { Position } \\
(\operatorname{arcsec})\end{array}$ \\
\hline \multicolumn{4}{|c|}{ Three-component Model ${ }^{\mathrm{b}}$} \\
\hline $23.7 \ldots$ & -14.1 & 4.7 & -0.025 \\
\hline $4.5 \ldots$ & 7.7 & 21.0 & 0 \\
\hline 40.2 & 32.4 & 4.0 & 0.025 \\
\hline \multicolumn{4}{|c|}{ Nine-component Model ${ }^{\mathrm{b}}$} \\
\hline $3.5^{\mathrm{c}} \ldots .$. & -21.6 & 4.5 & -0.0117 \\
\hline $23^{c} \ldots \ldots$ & -14.7 & 4.5 & -0.0210 \\
\hline $9^{c} \ldots$ & -10.6 & 4.7 & -0.0319 \\
\hline $3.5^{\mathrm{d}}$. & 0 & 5 & -0.0135 \\
\hline $3.5^{\mathrm{d}}$. & 9 & 5 & 0.0025 \\
\hline $3.5^{\mathrm{d}} \ldots \ldots$ & 18 & 5 & 0.0175 \\
\hline $9^{c} \ldots \ldots \ldots$ & 29 & 5.0 & 0.0355 \\
\hline $42^{\mathrm{c}} \ldots \ldots \ldots \ldots$ & 32.7 & 4.0 & 0.0260 \\
\hline 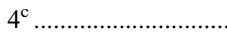 & 40 & 4.0 & 0.0162 \\
\hline
\end{tabular}

${ }^{\mathrm{a}}$ Widths $\left(\sigma_{v}\right)$ are in natural units $\left(\mathrm{FWHM}=2.3 \sigma_{v}\right)$. For a thermal line, $\sigma_{v}=$ $0.091 \sqrt{T_{e}}$, where $T_{e}$ is electron temperature. Hence for $T_{e}=10,000 \mathrm{~K}$, the thermal line width would be $9.1 \mathrm{~km} \mathrm{~s}^{-1}$, considerably broader than the model values of $4-5 \mathrm{~km} \mathrm{~s}^{-1}$. If, however, as suggested by Ponomarev et al. (1994), the masers are unsaturated with gain factures of about 5 , the lines will appear narrowed by a factor of $\sqrt{5}$, giving line widths of about $4 \mathrm{~km} \mathrm{~s}^{-1}$.

b The $p-v$ diagrams for these models are shown in Fig. 8.

c These components create the Keplerian wings in the $p$ - $v$ diagram. Their positions are appropriate for midline location, a central mass of $20 M_{\odot}$, a systemic velocity of $9 \mathrm{~km} \mathrm{~s}^{-1}$, and a central position of $0.0025^{\prime \prime}$.

$\mathrm{d}$ These three components create the central "gradient" in the $p-v$ diagram.

the centroid position within its velocity range of dominance. That is, the Gaussian rolloff of the outer components toward the center of the spectrum leaves the systemic velocity feature dominant at velocities near the systemic value. (Note that the "pulling" of the $p$ - $v$ diagram back toward zero at high velocities is simply the effect of the central feature's dominance at high velocities due to its uncharacteristically large line width.) This result shows that the approximately linear $p$ - $v$ diagram slope in the low-velocity region that we measured on MWC 349A cannot be explained by a simple two-component model or a more realistic three-component model described here. That is, the spectral separation of the outer components is sufficient to ensure that there are no "gradient" artifacts.

A more realistic model is one with nine components, whose parameters are listed in Table 1. The broad central component in the three-component model is replaced by three narrower components symmetrically placed in velocity and position, as shown in Figure 8. The purpose of these components is to create the observed gradient in the central region. In addition, the components at 32 and $-14 \mathrm{~km} \mathrm{~s}^{-1}$ have each been replaced by three components to simulate the Keplerian wings. These components follow Kepler's law (e.g., eq. [2], with $\theta=90^{\circ}$; i.e., midline position) for a stellar mass of $20 M_{\odot}$ and systemic velocity of $9 \mathrm{~km} \mathrm{~s}^{-1}$. As shown in Figure 8, this model fits the $p-v$ diagram and spectrum rather well. A key characteristic of this model is that the three components in each of the Keplerian wings are dominated by a strong component. In the centroid analysis, the strong component "pulls" the position of each of the weaker components so as to steepen the slope of the $p$ - $v$ diagram.

In the nine-component model we chose line widths of $4-$ $5 \mathrm{~km} \mathrm{~s}^{-1}$, which is reasonable for a thermal gas at $10^{4} \mathrm{~K}$, where an unsaturated maser gain of about 5 narrows the thermal line width by a factor of $\sqrt{5}$. However, a better model might be one of many 

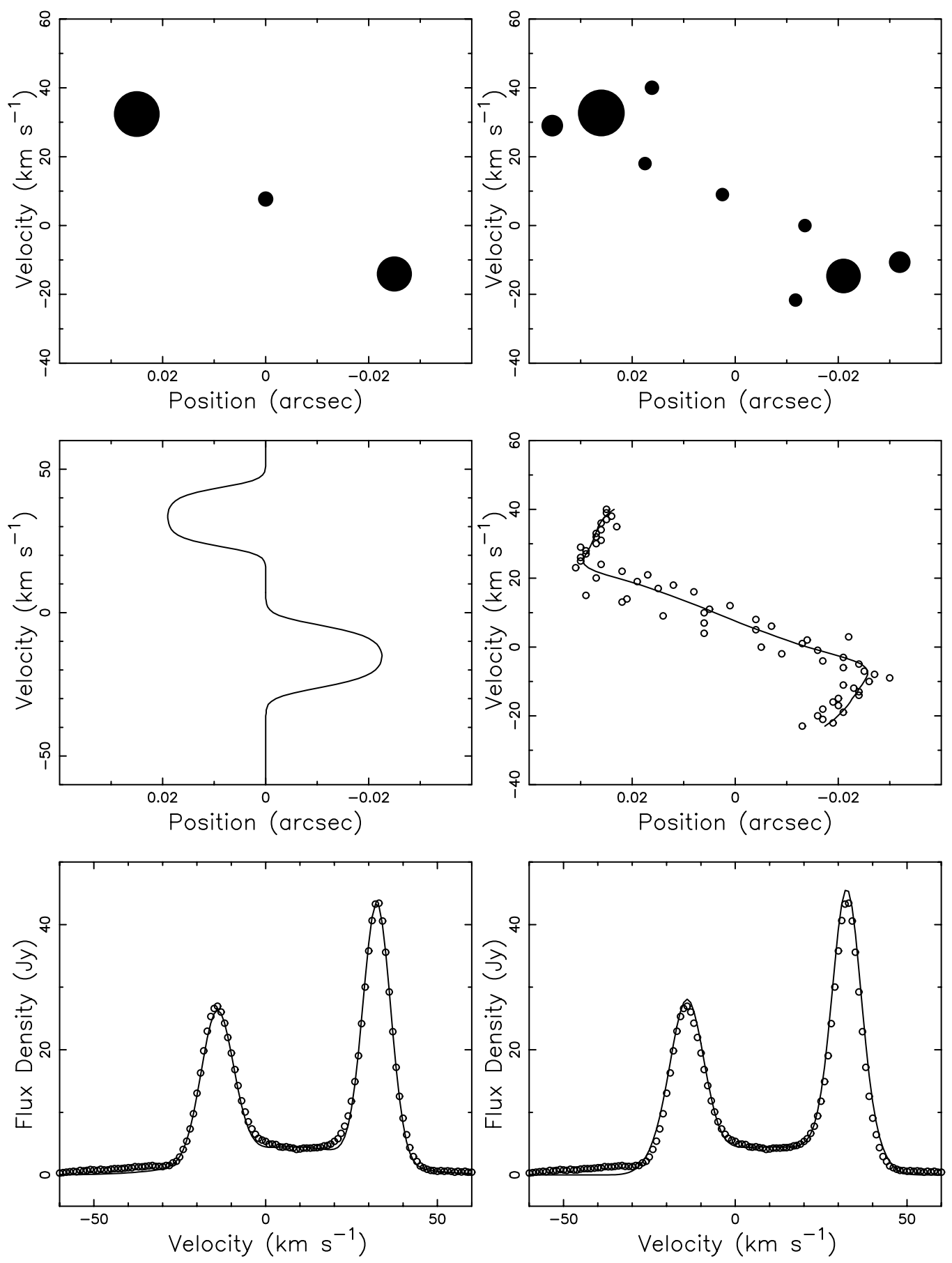

FIG. 8. - Two simulations of the maser distributions in MWC 349A from a model with components separated in only one spatial coordinate: three-spot model (left) and nine-spot model (right). The parameters of the maser distributions are listed in Table 1 and shown in the top panels (the areas of the spots are proportional to the flux density). The middle panels show the simulated $p$ - $v$ diagrams. In the middle right panel, the $p$ - $v$ diagram from the simulation is shown with the data (also shown in Fig. 4). In the bottom panels the simulated spectrum is plotted with the observed spectrum for each model. The nine-component model has 35 parameters. These parameters were not determined by least mean square fitting, but chosen to show an approximate fit and to demonstrate the characteristics of the model.

more components that are intrinsically much narrower (i.e., resulting from higher maser gain of $>100$ ) but add up to an approximately Gaussian profile of about $5 \mathrm{~km} \mathrm{~s}^{-1}$ width. In this case the spectral range of influence of each component will be relatively small, and the centroiding would not affect the $p-v$ diagram significantly. In this situation, the spiral arm model would be a viable interpretation of the data.
Instances of power-law indices in $p$ - $v$ diagrams of underresolved images of masers calculated from PCA analyses have been reported by others in the literature. Examples of these are the $\mathrm{CH}_{3} \mathrm{OH}$ maser emission in DR21(OH)N by Harvey-Smith et al. (2007) and the $\mathrm{SiO}$ maser in the late-type star R Aquarii by Cotton et al. (2004).

We note that W. J. Welch \& S. White (2007, private communication) have also imaged MWC 349A in the same transition 
with the BIMA array in its highest resolution mode of $0.1^{\prime \prime}$. Their image is very similar to ours, with a gradient at low velocities and Keplerian wings, although the centroiding is less accurate because of the somewhat lower $\mathrm{S} / \mathrm{N}$. Although their resolution was still insufficient to resolve the masers, we are greatly encouraged that our results are consistent. In addition, Martín-Pintado \& Thum (2007) presented maser images with the Plateau-de-Bure interferometer at a recent conference, which clearly shows the linear structure of the systemic features. They also measured features beyond our range $\left(>42,<-21 \mathrm{~km} \mathrm{~s}^{-1}\right)$, which are offset from the linear structure and may be due to a wind component. As can be seen in Figure 8 (lower right panel) our spectrum shows significant power beyond 40 and $-21 \mathrm{~km} \mathrm{~s}^{-1}$ that was too weak for us to find accurate positions for, which could be part of a wind component.

\section{CONCLUSIONS}

We have presented a new high-resolution image of the maser features in the inner ionized envelop of MWC 349A. The masers act as tracers of the velocity distribution in the disk. We used the PCA method to determine the positions of the maser spots to an accuracy much finer than the resolution of the interferometer. Based on our simulations we find that there are two plausible interpretations to our data. In both models the linear distribution of masers in the $p-v$ diagram between -10 and $25 \mathrm{~km} \mathrm{~s}^{-1}$ suggests the presence of an annular ring of maser emission with a radius of about $0.025^{\prime \prime}$. The scatter is due to measurement noise, intrinsic thickness in the annular masing region, and inclination of the ring. If the high-velocity maser components have line widths of about $5 \mathrm{~km} \mathrm{~s}^{-1}$, then the simulations of the PCA method indicate that they may lie along the midline. If, on the other hand, these components have much narrower intrinsic line widths because of higher maser amplification, then they may be distributed along spiral structures.

If the maser components are the result of the unsaturated amplification, then their brightness temperatures will be much greater than the thermal temperature of the gas by a factor of $\exp (G)$, where $G$ is the maser gain factor. Thus, these masers may be observable at much higher resolution. ALMA (the Atacama Large Millimeter Array), in its extended configuration of $10 \mathrm{~km}$, could be used to observe MWC 349 (which will have a maximum elevation angle of about $30^{\circ}$ ) at a resolution of about $0.02^{\prime \prime}$ at $230 \mathrm{GHz}$, and could distinguish between the models we have proposed.

We thank Henrik Beuther, Chunhua Qi, David Fong, Mark Gurwell, and Jun-Hui Zhao for help with the preparation of the observing script as well as the data calibration and reduction. We are grateful for the astute remarks of the anonymous referee, who urged us to look more deeply into the simulations reported here and made other very useful suggestions. We thank William Danchi for providing digital data for Figures 1 and 4, and Yolanda Gómez and Daniel Tofoya for providing the digital image for Figure 1. Jack Welch encouraged us to carry out a simulation of centroiding analysis and showed us the BIMA images of MWC 349A, and Vladimir Strelnitski discussed radiative transfer issues and dynamical models with us. We also benefited from discussions with Naama Dror, Yolanda Gómez, Lisa Harvey-Smith, Luis Rodríguez, Howard Smith, and Daniel Tafoya.

Facility: SMA
Buta, R. J., Byrd, G. G., \& Freeman, T. 2003, AJ, 125, 634

Chen, H.-R., Welch, W. J., Wilner, D. J., \& Sutton, E. C. 2006, ApJ, 639, 975

Cohen, M., Bieging, J. H., Dreher, J. W., \& Welch, W. J. 1985, ApJ, 292, 249

Cotton, W. D., et al. 2004, A\&A, 414, 275

Danchi, W. C., Tuthill, P. G., \& Monnier, J. D. 2001, ApJ, 562, 440

Gordon, M. A. 2003, ApJ, 589, 953

Gordon, M. A., Holder, B. P., Jisonna, L. J., Jorgenson, R. A., \& Strelnitski, V. S. 2001, ApJ, 559, 402

Harvey-Smith, L., Soria-Ruiz, R., Duarte-Cabral, A., \& Cohen, R. J. 2008, MNRAS, 384, 719

Hollenbach, D., Johnstone, D., Lizano, S., \& Shu, F. 1994, ApJ, 428, 654

Kohoutek, L., \& Wehmeyer, R. 1999, A\&AS, 134, 255

Martín-Pintado, J., \& Thum, C. 2007, Transformational Science with ALMA (Charlottesville: NRAO), http://www.cv.nrao.edu/naasc/disk07/talks / JMartinPintado.pdf

Martín-Pintado, J., Thum, C., \& Bachiller, R. 1989, A\&A, 222, L9

Moran, J. M., Greenhill, L. J., \& Herrnstein, J. R. 1999, J. Astrophys. Astron., 20,165
Planesas, P., Martín-Pintado, J., \& Serabyn, E. 1992, ApJ, 386, L23

Ponomarev, V. O., Smith, H. A., \& Strelnitski, V. S. 1994, ApJ, 424, 976

Quillen, A. C., Varnière, P., Minchev, I., \& Frank, A. 2005, AJ, 129, 2481

Reid, M. J., Haschick, A. D., Burke, B. F., Moran, J. M., Johnston, K. J., \& Swenson, G. W., Jr. 1980, ApJ, 239, 89

Rodríguez, L. F., \& Bastian, T. S. 1994, ApJ, 428, 324

Rodríguez, L. F., Gómez, Y., \& Tafoya, D. 2007, ApJ, 663, 1083

Strelnitski, V., Haas, M. R., Smith, H. A., Erickson, E. F., Colgan, S. W. J., \& Hollenbach, D. J. 1996a, Science, 272, 1459

Strelnitski, V., Ponomarev, V. O., \& Smith, H. A. 1996b, ApJ, 470, 1118

Tafoya, D., Gómez, Y., \& Rodríguez, L. F. 2004, ApJ, 610, 827

Thum, C., Matthews, H. E., Harris, A. I., Tacconi, L. J., Schuster, K. F., \& Martín-Pintado, J. 1994a, A\&A, 288, L25

Thum, C., Matthews, H. E., Martín-Pintado, J., Serabyn, E., Planesas, P., \& Bachiller, R. 1994b, A\&A, 283, 582

Thum, C., \& Morris, D. 1999, A\&A, 344, 923

White, R. L., \& Becker, P. H. 1985, ApJ, 297, 677 